\title{
Ursodeoxycholic Acid and S-adenosylmethionine for the Treatment of Intrahepatic Cholestasis of Pregnancy: A Meta-analysis
}

\author{
Yang Zhang, ${ }^{1,2}$ Linlin Lu, ${ }^{3,4}$ David W Victor, ${ }^{5}$ Yongning Xin,,${ }^{1,2,3,}$ and Shiying Xuan ${ }^{1,2,3,{ }^{*}}$ \\ ${ }^{1}$ Medical College, Qingdao University, Qingdao, China \\ ${ }^{2}$ Department of Gastroenterology, Qingdao Municipal Hospital, Qingdao, China \\ ${ }^{3}$ Digestive Disease Key Laboratory of Qingdao, Qingdao, China \\ ${ }^{4}$ Central Laboratories, Qingdao Municipal Hospital, Qingdao, China \\ ${ }^{5}$ Hepatology and Transplant Medicine, Department of Medicine, Houston Methodist Hospital, Houston, USA \\ "Corresponding authors: Shiying Xuan, Department of Gastroenterology, Qingdao Municipal Hospital, Qingdao, Shandong Province, China. Tel: +86-53288905508, Fax: \\ +86-53288905293, E-mail: xuansydxy@163.com; Yongning Xin, Department of Gastroenterology, Qingdao Municipal Hospital, Qingdao, Shandong Province, China. Tel: \\ +86-53282789463, Fax: +86-53285968434, E-mail: xinyongning@163.com
}

Received 2016 April 17; Revised 2016 June 22; Accepted 2016 June 28.

\begin{abstract}
Context: An optimal therapeutic strategy has not yet been identified for the pharmacological treatment of intrahepatic cholestasis of pregnancy (ICP). The aim of this study was to evaluate the efficacy and safety of ursodeoxycholic acid (UDCA) and Sadenosylmethionine (SAMe) in the treatment of ICP, both individually and in combination.

Evidence Acquisition: A meta-analysis of all randomized controlled trials (RCTs) comparing UDCA, SAMe, and combination therapy was performed. We carried out a literature search using pubmed, embase, the cochrane register of controlled trials, and the science citation index of web of science. The maternal clinical and biochemical responses, including pruritus scores, total bilirubin, total bile acids, alanine aminotransferase, and aspartate transaminase, were evaluated. Safety assessments, including preterm delivery, cesarean section, and meconium-stained amniotic fluid, were also analyzed.

Results: Five RCTs including 311 patients were evaluated. In comparison to SAMe, UDCA significantly reduced the pruritus score (OR $=-0.45,95 \%$ confidence interval [CI]: -0.66 to $-0.25, \mathrm{P}<0.0001$ ) and improved the levels of total bile acids (TBAs; OR = $-0.59,95 \% \mathrm{CI}$ : -0.99 to $-0.30, \mathrm{P}<0.0001$ ) and alanine aminotransferase (ALT; OR $=-0.38,95 \% \mathrm{CI}$ : -0.66 to $-0.09, \mathrm{P}=0.01$ ). UDCA was associated with significantly lower preterm delivery rates than SAMe $(\mathrm{RR}=0.48,95 \% \mathrm{CI}$ : $0.32-0.72, \mathrm{P}=0.0004)$. Interestingly, combination therapy significantly reduced total bilirubin (TB; vs. SAMe, $\mathrm{OR}=-0.41,95 \% \mathrm{CI},-0.74$ to $-0.08, \mathrm{P}=0.02$ ), aspartate transaminase (AST; vs. UDCA, $\mathrm{OR}=-0.40,95 \% \mathrm{CI},-0.74$ to $-0.06, \mathrm{P}=0.02$ ), and the rate of preterm delivery (vs. SAMe, $\mathrm{OR}=0.62,95 \% \mathrm{CI}, 0.42-0.91, \mathrm{P}=0.02$ ), in comparison with either drug administered alone.

Conclusions: UDCA decreased the pruritus score, TBA, and ALT levels more effectively than SAMe, reducing the rate of preterm delivery for ICP.
\end{abstract}

Keywords: Ursodeoxycholic Acid, S-Adenosylmethionine, Intrahepatic Cholestasis of Pregnancy

\section{Context}

Intrahepatic cholestasis of pregnancy (ICP) is a unique hepatic disorder in pregnancy characterized by mild to severe pruritus and disturbed liver function (1-6). ICP is a reversible form of cholestasis occurring mainly in the late second or third trimester of pregnancy, and tends to rapidly resolve after delivery $(3,7,8)$. The etiology of ICP is multifactorial and poorly understood; it may be triggered by the cholestatic effects of pregnancy hormones and their metabolites in genetically predisposed women. Multiple factors have been implicated in the pathogenesis of ICP, including environmental influences, nutritional deficiencies, hormonal changes, and genetic variations (9). Although ICP is usually associated with favorable pregnancy outcomes, it may seriously affect the fetus, and it is associated with complications such as premature delivery, meconium-stained amniotic fluid, fetal distress, sudden intrauterine fetal death, stillbirth, and even neonatal death. Thus, women with ICP should be considered high-risk, and the fetus should be carefully monitored during the third trimester. Pharmacological treatment of ICP aims to reduce the maternal symptoms and prevent fetal distress or sudden fetal death (10); however, an optimal therapeutic strategy has not yet been identified. Clinical trials and observational studies conducted over the last 20 years have indicated that ursodeoxycholic acid (UDCA) and S-adenosylmethionine (SAMe) can improve pruritus and serum biochemical abnormalities, further improving perinatal outcomes (11-16). UDCA is a hydrophilic bile acid that detoxifies hydrophobic bile acids, preventing injury to the bile ducts. SAMe is the principal glutathione precur- 
sor and methyl group donor involved in the synthesis of phosphatidylcholine. SAMe not only influences the composition and fluidity of hepatocyte plasma membranes, it also increases the methylation and biliary excretion of hormone metabolites (10). Two previous studies have shown that UDCA and SAMe may have synergistic effects due to their different biochemical mechanisms (17-19). A study by Zhou et al. focused on comparing the effects of UDCA, SAMe, and UDCA + SAMe on the rates of Cesarean section, preterm birth, fetal asphyxia, amniotic fluid pollution, and neonatal weight, but not on the maternal clinical and biochemical responses (20). Therefore, we carried out this meta-analysis to evaluate and compare the efficacy and safety of UDCA and SAMe for the treatment of ICP.

\section{Evidence Acquisition}

\subsection{Identification and Selection of Studies}

Relevant studies were identified and selected by searching PubMed (updated to Nov 2015), Embase (1980November 2015), the cochrane register of controlled trials (Cochrane Library Issue 4, 2015), and the science citation index of web of science (1981-November 2015). The key search terms were "intrahepatic cholestasis of pregnancy," “cholestasis," "pregnancy," "ursodeoxycholic acid," "UDCA," "S-adenosylmethionine," and "SAMe" either alone or in combination with the terms "randomized controlled clinical trials" or "clinical trials". Only English language RCTs were accepted.

The following selection criteria were applied: 1) Study design: randomized or untreated controlled trial; 2) Study population: patients with ICP according to the RCOG green-top guidelines for obstetric cholestasis (21); and 3) Patients received either UDCA, SAMe, or a combination of both throughout the duration of the trial. The decision to include any trial was made by two researchers independently (ZY and YX), and any disagreements were resolved by discussion.

\subsection{Study Objective and Definition of End Points}

We selected the following eight clinically meaningful parameters to estimate the efficacy and safety of treatment with UDCA, SAMe, or a UDCA + SAMe combination: 1) Pruritus score, 2) Total bile acid (TBA), 3) Total bilirubin (TB), 4) Alanine aminotransferase (ALT), 5) Aspartate transaminase (AST), 6) Preterm delivery, 7) Cesarean section, and 8) Meconium-stained amniotic fluid.

\subsection{Quality of Methodology}

The methodological quality of the included studies was scored with the Jadad composite scale (Box 1) (22, 23), a five-point quality scale. According to this scale, studies with a score of $\leq 2$ are considered low-quality, while those with a score of $\geq 3$ are considered high-quality (23, 24). The methodological quality was assessed by two of the authors of the present study (ZY and YX). Each study was given an overall quality score based on the above criteria, then ranked accordingly. Any disagreement was resolved by consensus.

\subsection{Statistical Methods}

Data was analyzed using the Mantel-Haenszel method (fixed-effect model) or the DerSimonian and Laird method (random-effects model) with the meta-analysis software review manager (RevMan 5.3, Cochrane Collaboration, Oxford, England) $(25,26)$. The odds ratio (OR) for each clinical event was presented with a $95 \%$ confidence interval (CI). Heterogeneity between the trials was tested with $\chi^{2}$ tests, with a P value of 0.05 indicating significant heterogeneity. The OR for each clinical event was pooled with the fixedeffect model, and if the $\chi^{2}$ test for heterogeneity was significant, the analysis was also carried out using the randomeffects model.

\section{Results}

\subsection{Description of the Selected Studies}

A total of 35 studies were identified using our search strategy. Among these studies, we eliminated those that did not fulfill the meta-analysis inclusion criteria, and identified a total of five RCTs comparing UDCA with SAMe (Table 1) (17-19, 27, 28). In one study, a placebo group was used in addition to the UDCA, SAMe, and UDCA + SAMe groups (17). From this study, we included only the data on the UDCA, SAMe, and UDCA + SAMe groups in our analysis. All of the included studies had been published as peerreviewed articles. The main characteristics of these five RCTs are listed in Table 1.

\subsection{Maternal Clinical and Biochemical Response}

Two studies $(17,19)$ provided the pruritus scores of the patients; four $(17-19,28)$ provided the serum TB, TBA, and ALT levels; and three $(18,19,28)$ provided the serum AST levels before and after commencement of treatment. The study by Floreani et al. (27), however, did not provide maternal clinical and biochemical response data. 
Box 1. Criteria for Grading the Quality of Randomized Controlled Trials: Jadad Score

\begin{tabular}{|c|c|c|c|c|c|c|c|}
\hline \multicolumn{8}{|c|}{ Study Received a Score of 1 for Each "Yes" Response and o for Each "No" Response for Each of th } \\
\hline \multicolumn{8}{|c|}{ 1. Was the study described as randomized using the words randomly, random, and randomization? } \\
\hline \multicolumn{8}{|c|}{ a) An additional point was given if the method of randomization was described and was appropriate (e.g., table of random numbers, computer-generated). } \\
\hline \multicolumn{8}{|c|}{ b) A point was deducted if the reported method of randomization was inappropriate (e.g., patients allocated alternately, by birth date, or hospital number). } \\
\hline \multicolumn{8}{|c|}{ 2. Was the study described as double-blinded? } \\
\hline \multicolumn{8}{|c|}{ a) A point was given if the method of blinding was described and was appropriate (e.g., identical placebo). } \\
\hline \multicolumn{8}{|c|}{ b) A point was deducted if an inappropriate method of blinding was reported (e.g., comparing placebo tablets with injection). } \\
\hline \multicolumn{8}{|c|}{ 3. Were the withdrawals and dropouts described? } \\
\hline \multicolumn{8}{|c|}{ A maximum of five points could be allocated per study. } \\
\hline \multicolumn{8}{|c|}{ Table 1. Characteristics of the Selected Randomized Controlled Trials } \\
\hline Study, y & Country & $\begin{array}{l}\text { No. of Patients } \\
\text { (UDCA/SAMe/UDCA } \\
\text { +SAMe) }\end{array}$ & UDCA Dose, mg/d & SAMe Dose, mg/d & $\begin{array}{l}\text { UDCA + SAMe } \\
\text { Dose, } \mathbf{m g} / \mathbf{d}\end{array}$ & $\begin{array}{c}\text { Planned } \\
\text { Treatment } \\
\text { Duration }\end{array}$ & $\begin{array}{l}\text { Jadad Quality } \\
\quad \text { Score }\end{array}$ \\
\hline $\begin{array}{l}\text { Floreani (27) } \\
1996\end{array}$ & Italy & $\begin{array}{l}10 / 10 / \text { Not } \\
\text { reported }\end{array}$ & 450 & 1000 & Not reported & Until delivery & 3 \\
\hline Nicastri (17) 1998 & Italy & $8 / 8 / 8$ & 600 & 1000 & $600+1000$ & 20 days & 3 \\
\hline $\begin{array}{l}\text { Roncaglia (28) } \\
2004\end{array}$ & Italy & $\begin{array}{l}24 / 22 / \text { Not } \\
\text { reported }\end{array}$ & 600 & 800 & Not reported & Until delivery & 4 \\
\hline Binder (18) 2006 & Czech & $26 / 25 / 27$ & 750 & 1000 & $750+1000$ & Until delivery & 3 \\
\hline Zhang (19) 2015 & China & $41 / 38 / 41$ & 1000 & 1000 & $1000+1000$ & 14 days & 3 \\
\hline
\end{tabular}

\subsubsection{Pruritus Score}

Inter-trial heterogeneity was found in pruritus scores between UDCA + SAMe vs UDCA and UDCA + SAMe vs. SAMe (UDCA + SAMe vs. UDCA, P = 0.001, I2 = 91\%; UDCA + SAMe vs. SAMe, $\mathrm{P}=0.009, \mathrm{I} 2=85 \%)$, so the random-effects model was used. No heterogeneity was found between the UDCA vs. SAMe treatments $(\mathrm{P}=0.40, \mathrm{I} 2=0 \%)$; in this case, therefore, a fixed-effects model was used. The meta-analysis demonstrated that the pruritus scores were significantly lower in the UDCA group than in the SAMe group after the treatment $(\mathrm{OR}=-0.45,95 \% \mathrm{CI},-0.66$ to $-0.25, \mathrm{P}<0.0001)$. However, the pruritus scores did not differ significantly between the UDCA + SAMe and UDCA treatment groups $(\mathrm{OR}=$ $0.32,95 \% \mathrm{CI},-0.63$ to $1.27, \mathrm{P}=0.51)$ or the UDCA + SAMe and SAMe groups $(\mathrm{OR}=-0.07,95 \% \mathrm{CI},-0.80$ to $0.67, \mathrm{P}=0.86$ ) (Figure 1).

\subsubsection{Total Bilirubin}

No heterogeneity was found in TB between UDCA and SAMe, UDCA + SAMe and UDCA, and UDCA + SAMe and SAMe (UDCA vs. SAMe, $\mathrm{P}=0.88, \mathrm{I} 2=0 \%$; UDCA+SAMe vs UDCA, $\mathrm{P}=0.41, \mathrm{I} 2=0 \%$; UDCA+SAMe vs. SAMe, $\mathrm{P}=0.19$, I2 $=39 \%$ ); therefore, a fixed-effects model was used. The metaanalysis demonstrated that after the treatment, serum TB levels were significantly lower in the UDCA + SAMe group than in the SAMe group ( $\mathrm{OR}=-0.41,95 \% \mathrm{CI},-0.74$ to -0.08 , $\mathrm{P}=0.02)$. However, serum TB levels did not differ significantly between the UDCA and SAMe groups ( $O R=-0.25,95 \%$ CI, -0.53 to $0.03, \mathrm{P}=0.08)$ or the UDCA + SAMe and UDCA groups $(\mathrm{OR}=-0.19,95 \% \mathrm{CI},-0.51$ to $0.13, \mathrm{P}=0.25)$ (Figure 2 ).

\subsubsection{Total Bile Acids}

For TBA, inter-trial heterogeneity was found between the UDCA + SAMe and UDCA groups and the UDCA + SAMe and SAMe groups (UDCA + SAMe vs. UDCA, $\mathrm{P}=0.02, \mathrm{I} 2=$ $75 \%)$ and between the UDCA + SAMe vs. SAMe groups ( $\mathrm{P}=$ $0.0002, \mathrm{I} 2=88 \%$ ); therefore, the random-effects model was used. No heterogeneity was found between the UDCA and SAMe groups $(\mathrm{P}=0.13, \mathrm{I} 2=47 \%)$, and a fixed-effects model was used. The meta-analysis demonstrated that after the treatment, serum TBA levels were significantly lower in the UDCA group than in the SAMe group $(\mathrm{OR}=-0.52,95 \% \mathrm{CI}$, 0.81 to $-0.23, \mathrm{P}=0.0005)$. However, serum TBA levels did not differ significantly between the UDCA + SAMe and UDCA treatment groups $(\mathrm{OR}=0.07,95 \% \mathrm{CI},-0.60$ to $0.74, \mathrm{P}=0.84)$ or between the UDCA+SAMe and SAMe treatment groups $(\mathrm{OR}=-0.44,95 \% \mathrm{CI},-1.56$ to $0.68, \mathrm{P}=0.44)$ (Figure 3$)$. 
A

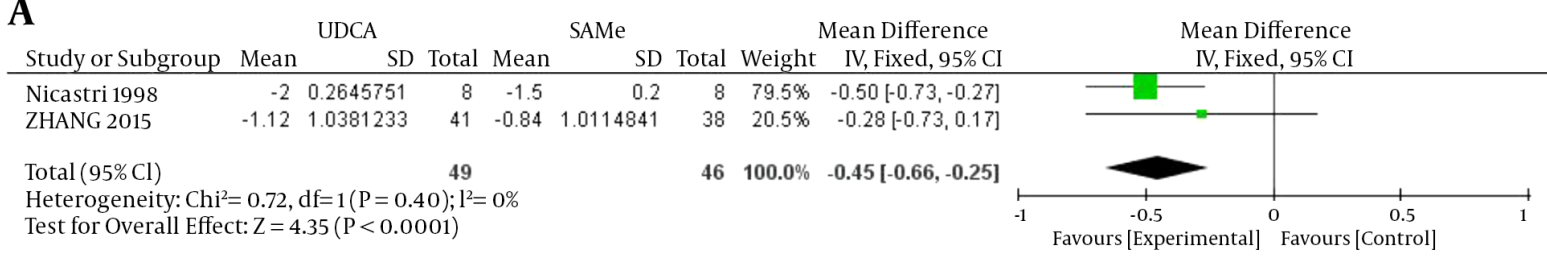

B

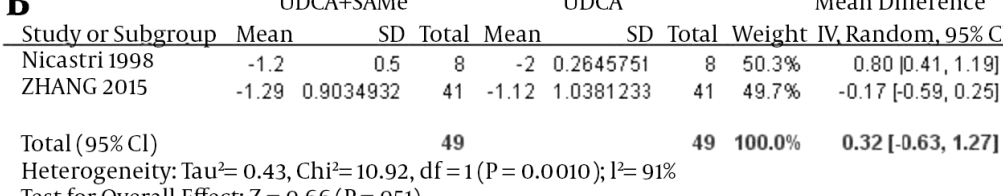

Heterogeneity: $\mathrm{Tau}^{2}=0.43, \mathrm{Chi}^{2}=10.92, \mathrm{df}=1(\mathrm{P}=0.0010) ; \mathrm{l}^{2}=91 \%$

Test for Overall Effect: $\mathrm{Z}=0.66(\mathrm{P}=051)$

C $\begin{array}{lll}\text { UDCA+SAMe } & \text { SAMe } \\ \text { Study or Subgroup Mean } & \text { SD Total Mean }\end{array}$ $\begin{array}{lll}\text { Nicastri } 1998 & -1.2 & 0.5\end{array}$ ZHANG 2015

\begin{abstract}
$\begin{array}{lll}8 & 50.9 \% & 0.30[-0.07,0.67]\end{array}$
$38 \quad 49.1 \% \quad-0.45[-0.87,-0.03]$

$46100.0 \%$

$-0.07[-0.80,0.67]$
\end{abstract}

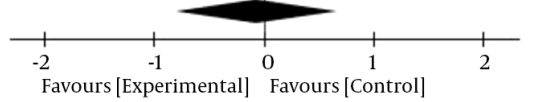

A, UDCA and SAMe; B, UDCA + SAMe and UDCA; C, UDCA + SAMe and SAMe.

Figure 2. Effects of UDCA, SAMe, and UDCA + SAMe on total bilirubin

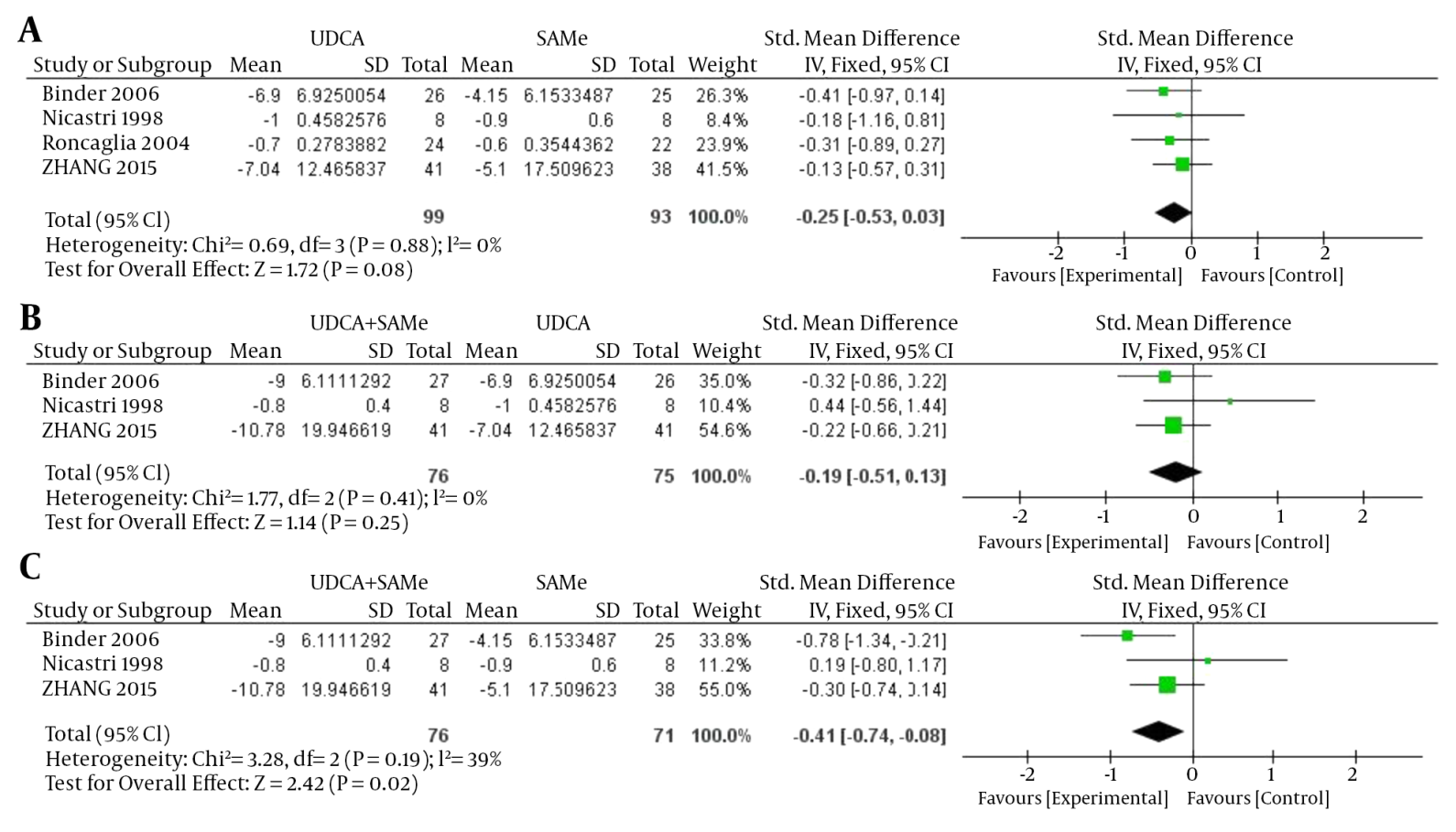




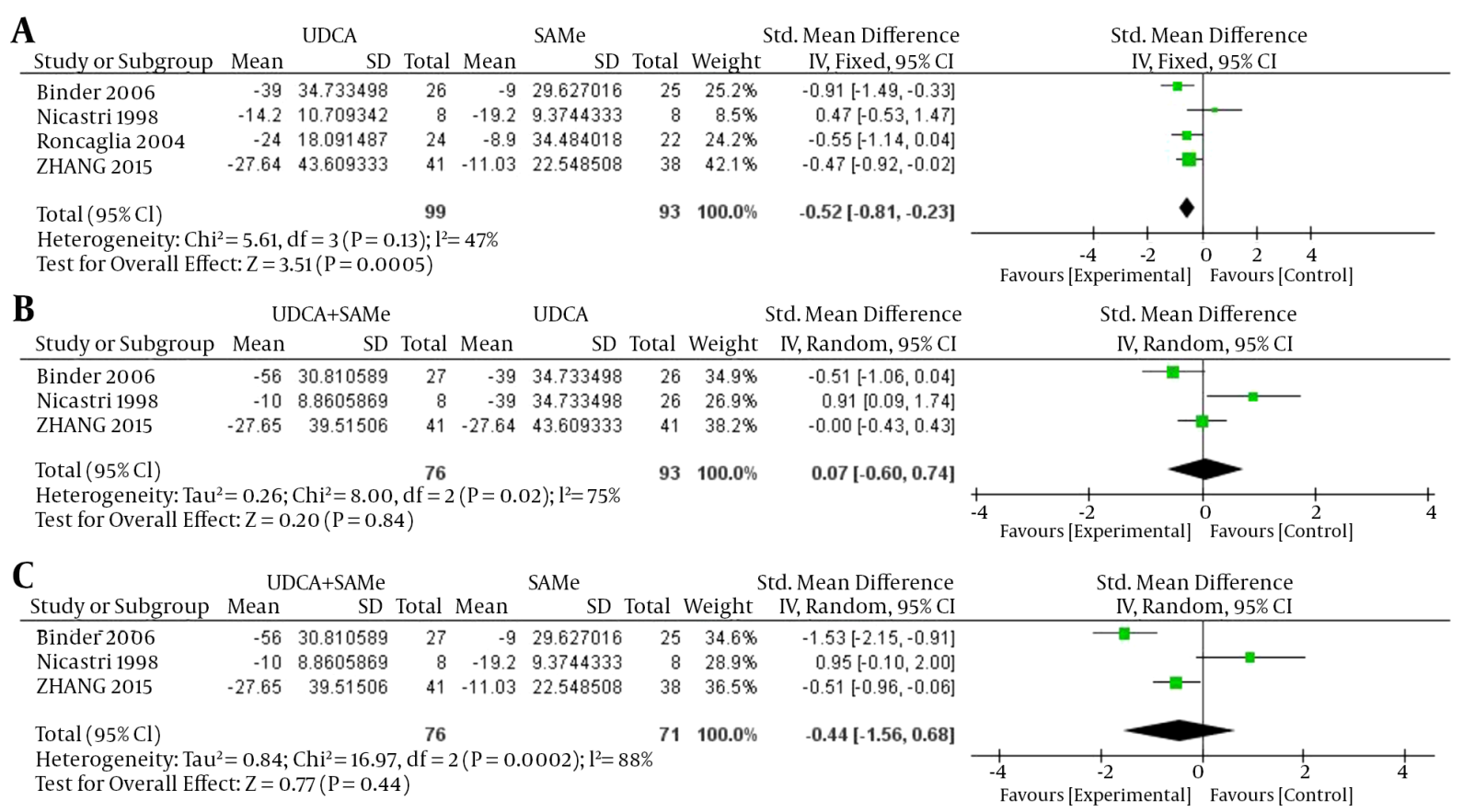

\subsubsection{Alanine Aminotransferase}

For ALT, inter-trial heterogeneity was found between the UDCA + SAMe and SAMe groups ( $\mathrm{P}=0.01, \mathrm{I} 2=78 \%$ ), so the random-effects model was used. No heterogeneity was found in the UDCA and SAMe ( $\mathrm{P}=0.32, \mathrm{I} 2=14 \%)$ and UDCA + SAMe and UDCA groups $(\mathrm{P}=0.33, \mathrm{I} 2=9 \%)$; therefore, a fixed-effects model was used. The meta-analysis demonstrated that after the treatment, serum ALT levels were significantly lower in the UDCA group than in the SAMe group $(\mathrm{OR}=-0.38,95 \% \mathrm{CI},-0.66$ to $-0.09, \mathrm{P}=0.01)$. However, ALT values did not differ significantly between the UDCA + SAMe and UDCA groups $(\mathrm{OR}=-0.24,95 \% \mathrm{CI},-0.56$ to $0.09, \mathrm{P}=0.15)$ or between the UDCA + SAMe and SAMe treatment groups $(\mathrm{OR}=-0.48,95 \% \mathrm{CI},-1.26$ to $0.31, \mathrm{P}=0.23)$ (Figure 4$)$.

\subsubsection{Aspartate Transaminase}

For AST, inter-trial heterogeneity was found between the UDCA and SAMe treatment groups $(\mathrm{P}=0.002, \mathrm{I} 2=84 \%)$ and between the UDCA + SAMe and SAMe treatment groups $(\mathrm{P}=0.0002, \mathrm{I} 2=93 \%)$; therefore, the random-effects model was used. No heterogeneity was found in AST levels between the UDCA + SAMe and UDCA treatment groups ( $\mathrm{P}=$ $0.78, \mathrm{I} 2=0 \%$; therefore, the fixed-effects model was used. The meta-analysis demonstrated that after the treatment, serum AST levels were significantly lower in the UDCA + SAMe group than in the UDCA group $(\mathrm{OR}=-0.40,95 \% \mathrm{CI}$, -0.74 to $-0.06, P=0.02)$. However, serum AST levels did not differ significantly between the UDCA and SAMe groups $(\mathrm{OR}=-0.70,95 \% \mathrm{CI},-1.49$ to $0.09, \mathrm{P}=0.08)$ or the UDCA + SAMe and SAMe treatment groups (OR $=-1.09,95 \% \mathrm{CI},-2.55$ to $0.36, \mathrm{P}=0.14$ ) (Figure 5).

\subsection{Safety Assessment}

Of the five studies included in this meta-analysis, four $(18,19,27,28)$ reported the rates of preterm delivery and cesarean section, while three $(18,19,28)$ reported the rates of meconium-stained amniotic fluid.

\subsubsection{Preterm Delivery}

No heterogeneity was found in the rates of preterm delivery between the UDCA and SAMe groups $(\mathrm{P}=0.83, \mathrm{I} 2=$ $0 \%)$, the UDCA + SAMe and UDCA groups $(\mathrm{P}=0.61, \mathrm{I} 2=0 \%)$, or the UDCA + SAMe and SAMe groups $(\mathrm{P}=0.74, \mathrm{I} 2=0 \%)$; therefore, a fixed-effects model was used for the analysis. The meta-analysis showed that after treatment, serum AST levels were significantly lower in the UDCA group than in the SAMe group $(\mathrm{OR}=0.48,95 \% \mathrm{CI}, 0.32-0.72, \mathrm{P}=0.0004)$, and lower in the UDCA + SAMe group than in the SAMe group 


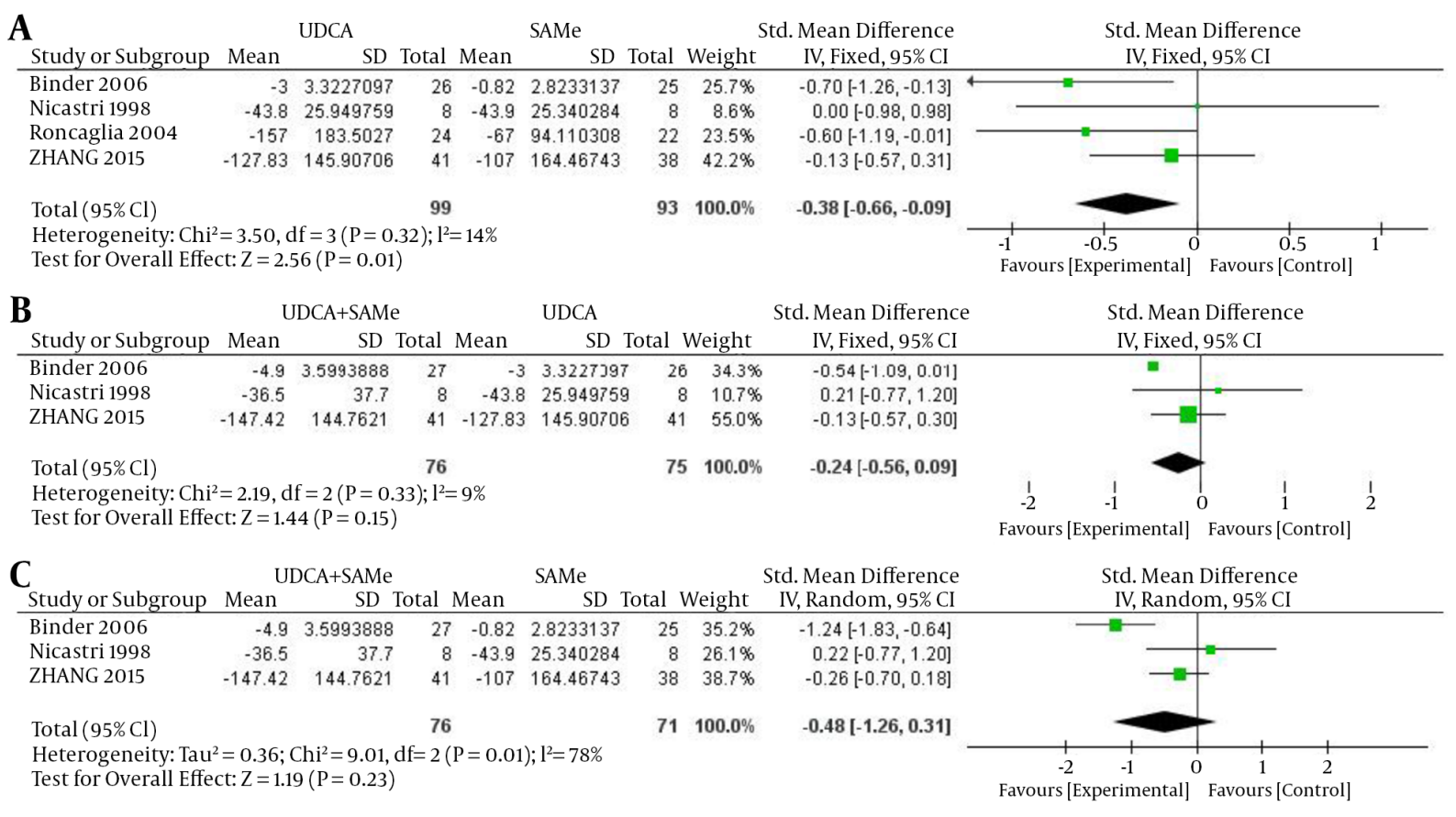

A, UDCA and SAMe, B, UDCA + SAMe and UDCA, C , UDCA + SAMe and SAMe.

Figure 5. Effects of UDCA, SAMe, and UDCA + SAMe on Aspartate Transaminase

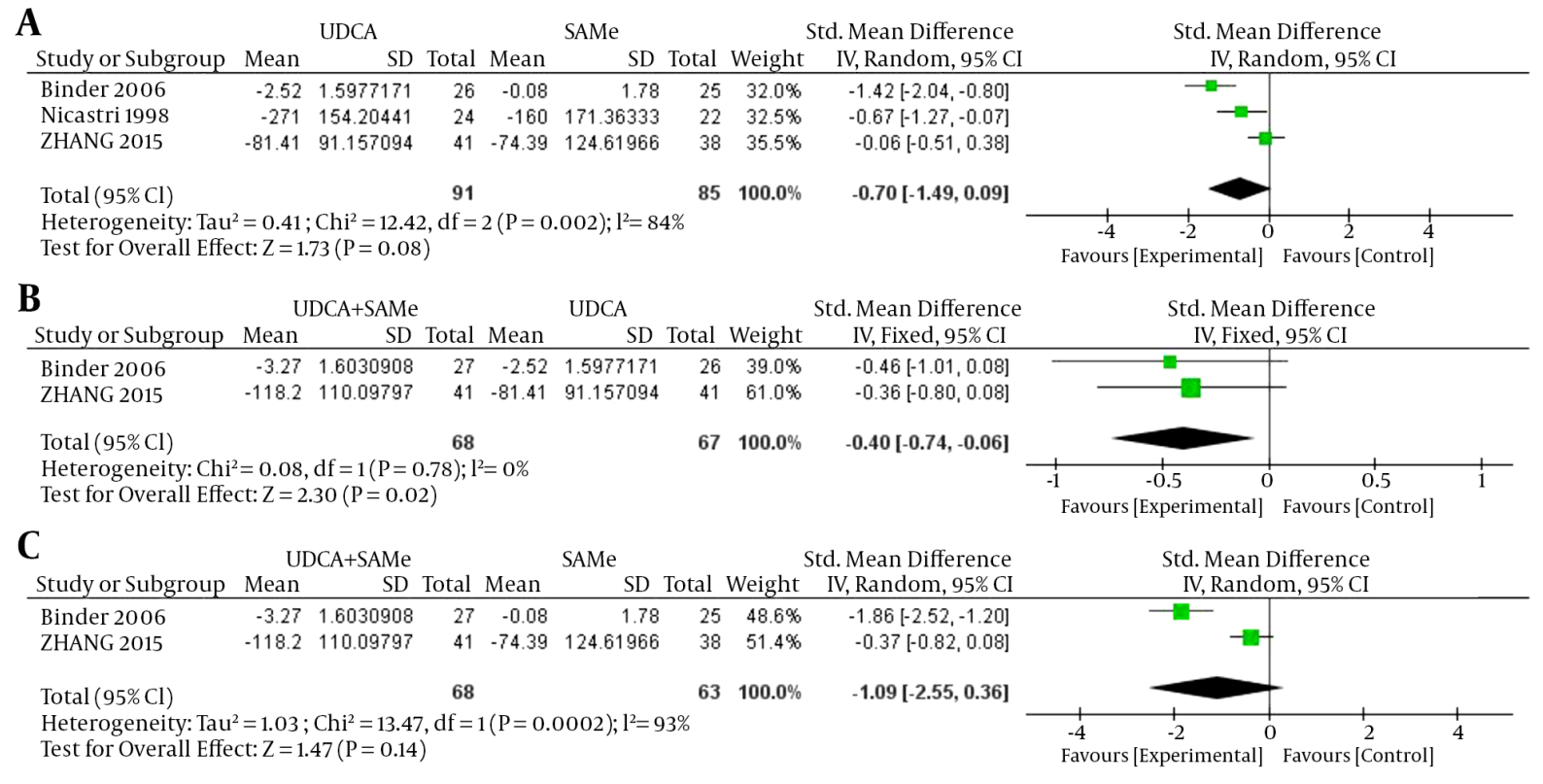


$(\mathrm{OR}=0.62,95 \% \mathrm{CI}, 0.42-0.91, \mathrm{P}=0.02)$. However, rates of preterm delivery did not differ significantly between the $\mathrm{UDCA}+$ SAMe and UDCA groups $(\mathrm{OR}=1.28,95 \% \mathrm{CI}, 0.76-2.16$, $\mathrm{P}=0.35$ ) (Figure 6).

\subsubsection{Cesarean Section}

No heterogeneity was found in the rates of cesarean section between the UDCA and SAMe groups $(\mathrm{P}=0.90, \mathrm{I} 2$ $=0 \%)$, the UDCA + SAMe and UDCA groups $(\mathrm{P}=0.54, \mathrm{I} 2=$ $0 \%)$, or the UDCA + SAMe and SAMe groups $(\mathrm{P}=0.23, \mathrm{I} 2=$ $30 \%$ ); therefore, a fixed-effects model was used. The metaanalysis demonstrated that after the treatment, there were no significant differences in the rates of cesarean section between the UDCA and SAMe groups ( $\mathrm{OR}=0.84,95 \% \mathrm{CI}$, $0.63-1.10, \mathrm{P}=0.20$ ), the UDCA + SAMe and UDCA groups (OR $=1.03,95 \% \mathrm{CI}, 0.75-1.42, \mathrm{P}=0.85)$, or the UDCA + SAMe and SAMe groups $(\mathrm{OR}=0.82,95 \% \mathrm{CI}, 0.61-1.09, \mathrm{P}=0.17)$ (Figure 7).

\subsubsection{Meconium-Stained Amniotic Fluid}

There was no heterogeneity in the rates of meconiumstained amniotic fluid between the UDCA and SAMe treatment groups $(\mathrm{P}=0.36, \mathrm{I} 2=1 \%)$, the UDCA + SAMe and UDCA groups $(\mathrm{P}=0.45, \mathrm{I} 2=0 \%)$, or the UDCA + SAMe and SAMe groups $(\mathrm{P}=0.93, \mathrm{I} 2=30 \%)$; therefore, a fixedeffects model was used. According to the meta-analysis, there were no significant differences between the UDCA and SAMe groups $(\mathrm{OR}=0.77,95 \% \mathrm{CI}, 0.40-1.46, \mathrm{P}=0.42)$, the UDCA + SAMe and UDCA groups $(\mathrm{OR}=0.82,95 \% \mathrm{CI}, 0.38-1.78$, $\mathrm{P}=0.62)$, or the UDCA + SAMe and SAMe groups $(\mathrm{OR}=0.77$, $95 \% \mathrm{CI}, 0.36-1.66, \mathrm{P}=0.51$ ) after treatment (Figure 8).

\section{Conclusions}

ICP is an uncommon occurrence in pregnancy, but is associated with adverse perinatal outcomes. As its pathogenesis is still not fully understood, the appropriate pharmacological treatment of ICP remains controversial. According to the latest EASL clinical practice guidelines for the management of cholestatic liver diseases (29), the goal of ICP treatment is not only to decrease itching and improve liver function, but also to improve pregnancy outcomes without any side effects for either the mother or the fetus. UDCA and SAMe have been used to treat ICP for decades (30). UDCA is a naturally-occurring hydrophilic bile salt that may increase the hydrophilic properties of the bile acid pool, thereby preventing damage to membranes by hydrophobic bile salts (31). SAMe influences methylation reactions, increasing the flow of bile and biliary lipid metabolism, which is impaired by the estrogen load produced by the placenta in patients with ICP (32).
To better explore the optimal treatment for ICP, we performed a meta-analysis to evaluate the efficacy and safety of UDCA and SAMe on maternal clinical and biochemical parameters, as well as fetal outcomes. To our knowledge, this is the first meta-analysis to comparatively analyze the use of UDCA and SAMe for ICP. Our analysis echoes previous reports showing that both drugs are effective and safe. One of the RCTs included in the present meta-analysis reported that UDCA and SAMe are both effective and safe in the treatment of ICP(19). UDCA has virtually no side effects, except for mild diarrhea in some cases. Because the start of treatment with UDCA is usually delayed until the third trimester, the risk of teratogenicity is further minimized (33).

The present meta-analysis included a total of eight clinical parameters (pruritus score, TBA, total bilirubin, ALT, AST, preterm delivery, cesarean section, and meconiumstained amniotic fluid) in order to assess the efficacy of UDCA and/or SAMe on ICP. Early onset of pruritus and high serum TBA levels were included as predictors of preterm delivery in ICP (1). Moreover, TB, ALT, and AST were also included, as they have been weakly correlated to preterm delivery (1).

Our results revealed that UDCA improves pruritus, TBA, and ALT more effectively than SAMe. Additionally, UDCA reduced the rate of preterm delivery more effectively than SAMe. Therefore, our analysis favors the use of UDCA as first-line therapy for ICP. Randomized trials have suggested that UDCA is a more effective therapy than SAMe for ICP (17$19,27,28)$. Roncaglia et al. reported that UDCA improved serum bile acid levels and other liver function tests more effectively than SAMe (28). However, UDCA and SAMe were equally effective at reducing pruritus. In contrast, Floreani et al. reported that UDCA and SAMe equally effectively controlled pruritus and TBA levels (27). In no study was UDCA inferior to SAMe.

As mentioned previously, these drugs have different modes of action and thus have the potential to be used synergistically. However, data regarding the concomitant use of these therapies is limited. Additionally, only a few studies have compared treatment of ICP with UDCA and SAMe. Binder et al. reported that while UDCA effectively treated ICP, combining it with SAMe produced a synergistic effect on the biochemical parameters. Although UDCA+SAMe was more effective than UDCA or SAMe alone, its effect on the fetal prognosis is unclear (18). Nicastri et al. also found that the combination of UDCA + SAMe was more effective than either drug used alone, and reported that UDCA + SAMe reduced pruritus and TB levels more effectively than UDCA alone (17), although levels of bile salts, alkaline phosphatase, and serum glutamic pyruvic transaminase did not differ significantly between the UDCA and UDCA 


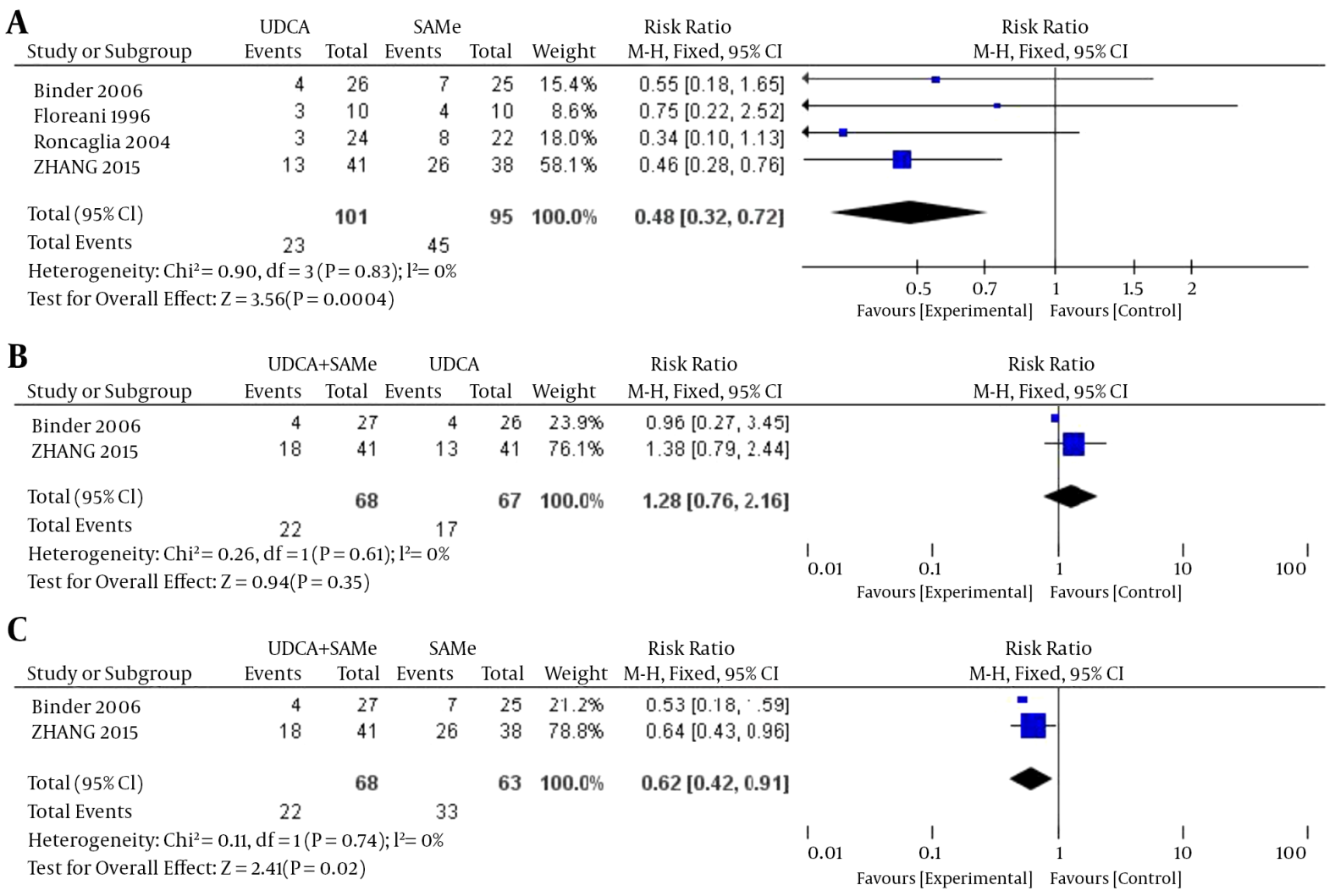

A,UDCA and SAMe; B, UDCA + SAMe and UDCA, C, UDCA + SAMe and SAMe.

+ SAMe groups. Furthermore, UDCA + SAMe could reduce bile salts and alkaline phosphatase more efficiently than SAMe alone. Zhang et al. indicated that UDCA monotherapy should be used as first-line therapy for ICP because it is more efficacious, cost-effective, and convenient (19). Our analysis echoes this recommendation.

The different efficacies of these two therapies may be attributed to their different pharmacological effects. Further studies should examine how both of these drugs influence ICP, as well as the differences in the mechanisms by which these therapies improve symptoms in ICP patients.

Interestingly, the results of this meta-analysis showed that while UDCA and SAMe alone had the aforementioned effects, they did not affect TB and AST levels. However, combination therapy significantly decreased these levels. We found that when applied together, these drugs may exert a synergistic effect in the treatment of ICP. Further studies should be carried out to study this effect.

In conclusion, this meta-analysis indicated that although both drugs are safe and relatively efficacious, UDCA monotherapy should be the first choice for the treatment of ICP, given that it was more effective than SAMe in reducing TBA, pruritus, and preterm delivery. Although both UDCA and SAMe have been used to treat ICP for several years, relatively few RCTs have compared the effects of these two drugs or a combination thereof. Our analysis suggested that compared with either drug administered alone, when used in combination, these drugs are more effective only in reducing AST (vs. UDCA), total bilirubin (vs. SAMe), and the rate of preterm delivery (vs. SAMe), and no evidence showed the combination to be better than either drug for the other parameters. It is not clear whether this synergistic relationship should be adopted for all cases of ICP or if SAMe can be added if biochemical or clinical parameters are not met after initiation of UDCA. Future studies should focus on optimized regimens of UDCA and SAMe, including the dosage and course, and whether they should be administered independently or in tandem. 
Figure 7. . Effects of UDCA, SAMe, and UDCA + SAMe on Rate of Cesarean Sections

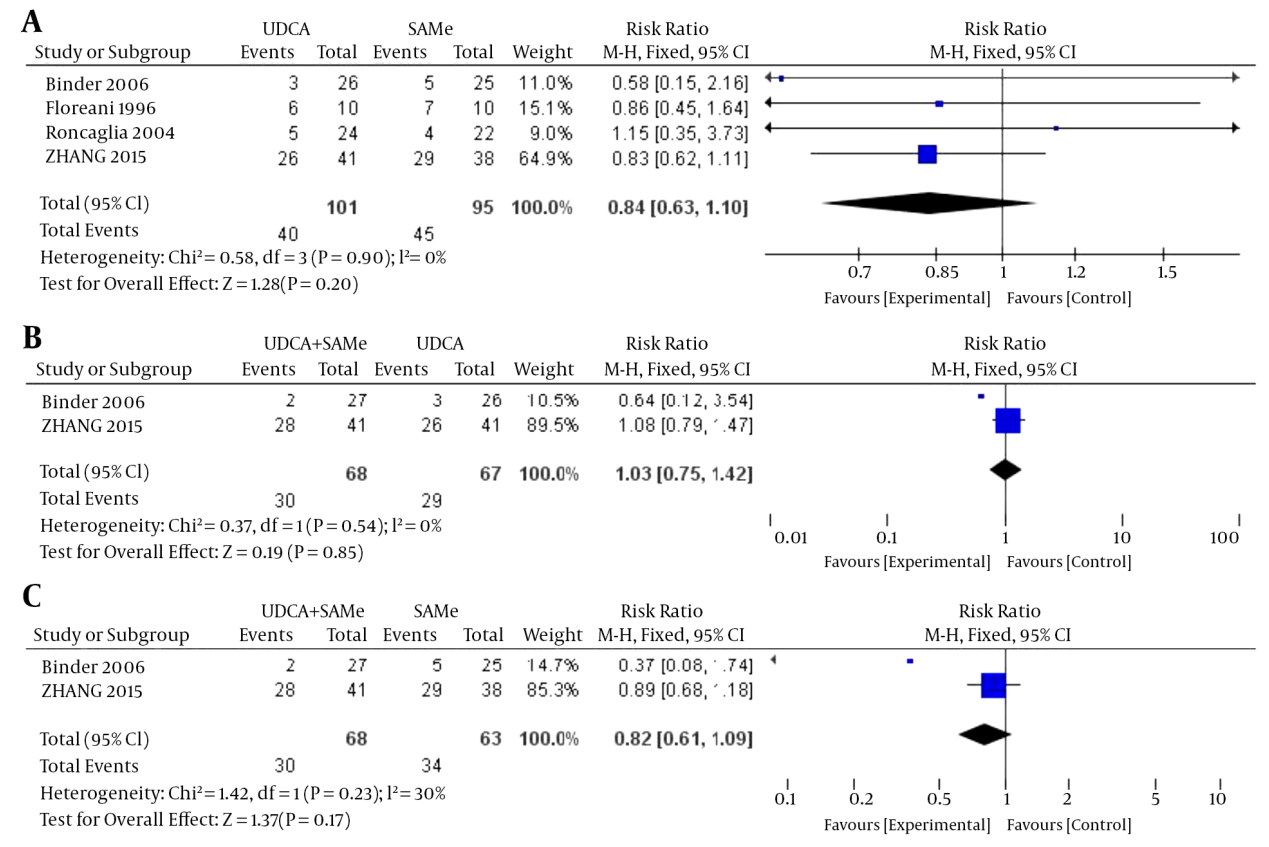

A, UDCA and SAMe; B, UDCA + SAMe and UDCA; C, UDCA + SAMe and SAMe.

Figure 8. Effects of UDCA, SAMe, and UDCA + SAMe on Rate of Meconium-Stained Amniotic Fluid

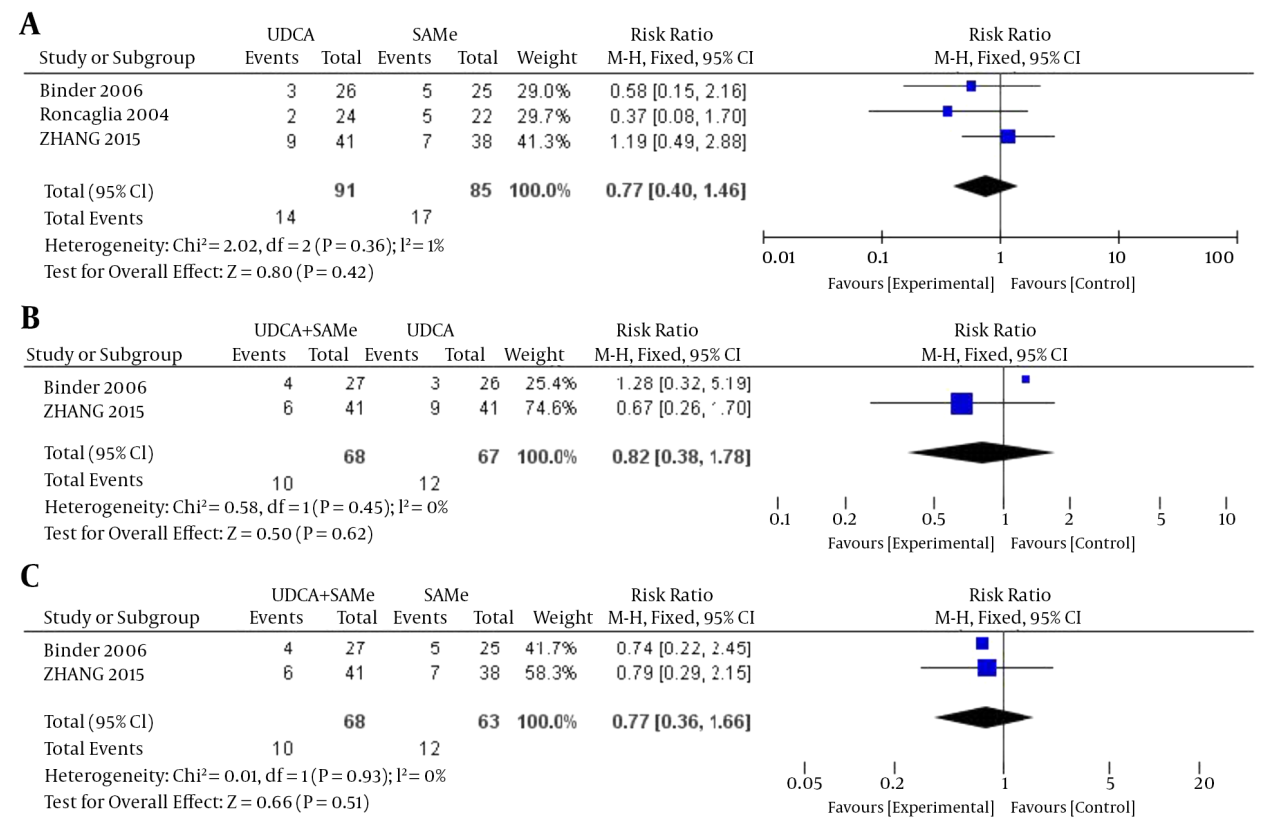




\section{Footnotes}

Authors' Contribution: Study concept and design: Yang Zhang, Linlin Lu, and Yongning Xin; acquisition of data: Yang Zhang, Linlin Lu, and Yongning Xin; analysis and interpretation of data: Yang Zhang and Yongning Xin; drafting of the manuscript: Yang Zhang, Linlin Lu, and Yongning Xin; critical revision of the manuscript for important intellectual content: David W. Victor and Shiying Xuan; statistical analysis: Yang Zhang; administrative, technical, and material support: David W. Victor and Yongning Xin; study supervision: Shiying Xuan.

Funding/Support: This study was supported by the Qingdao livelihood, science and technology project, China (Grant No.14-2-3-17-nsh) and the Qingdao key health discipline development fund.

\section{References}

1. Kondrackiene J, Kupcinskas L. Intrahepatic cholestasis of pregnancycurrent achievements and unsolved problems. World J Gastroenterol. 2008;14(38):5781-8. [PubMed: 18855975].

2. Arrese M, Reyes H. Intrahepatic cholestasis of pregnancy: a past and present riddle. Ann Hepatol. 2006;5(3):202-5. [PubMed: 17060884].

3. Lee NM, Brady CW. Liver disease in pregnancy. World J Gastroenterol. 2009;15(8):897-906. [PubMed: 19248187].

4. Ahmed KT, Almashhrawi AA, Rahman RN, Hammoud GM, Ibdah JA. Liver diseases in pregnancy: diseases unique to pregnancy. World J Gastroenterol. 2013;19(43):7639-46. doi: 10.3748/wjg.v19.i43.7639. [PubMed: 24282353].

5. Williamson C, Geenes V. Intrahepatic cholestasis of pregnancy. Obstet Gynecol. 2014;124(1):120-33. doi: 10.1097|AOG.0000000000000346. [PubMed: 24901263].

6. Gabzdyl EM, Schlaeger JM. Intrahepatic cholestasis of pregnancy: a critical clinical review. J Perinat Neonatal Nurs. 2015;29(1):41-50. doi: 10.1097/JPN.0000000000000077. [PubMed: 25633399].

7. Diken Z, Usta IM, Nassar AH. A clinical approach to intrahepatic cholestasis of pregnancy. Am J Perinatol. 2014;31(1):1-8. doi: 10.1055/s0033-1333673. [PubMed: 23359238].

8. Tan LK. Obstetric cholestasis: current opinions and management. Ann Acad Med Singapore. 2003;32(3):294-8. [PubMed: 12854371].

9. Pathak B, Sheibani L, Lee RH. Cholestasis of pregnancy. Obstet Gynecol Clin North Am. 2010;37(2):269-82. doi: 10.1016/j.ogc.2010.02.011. [PubMed: 20685553].

10. Ozkan S, Ceylan Y, Ozkan OV, Yildirim S. Review of a challenging clinical issue: Intrahepatic cholestasis of pregnancy. World J Gastroenterol. 2015;21(23):7134-41. doi: 10.3748/wjg.v21.i23.7134. [PubMed: 26109799].

11. Palma J, Reyes H, Ribalta J, Hernandez I, Sandoval L, Almuna R, et al. Ursodeoxycholic acid in the treatment of cholestasis of pregnancy: a randomized, double-blind study controlled with placebo. J Hepatol. 1997;27(6):1022-8. [PubMed: 9453428].

12. Liu Y, Qiao F, Liu H, Liu D. Ursodeoxycholic acid in the treatment of intraheptic cholestasis of pregnancy.J Huazhong Univ Sci Technolog Med Sci. 2006;26(3):350-2. [PubMed: 16961291].

13. Zapata R, Sandoval L, Palma J, Hernandez I, Ribalta J, Reyes H, et al. Ursodeoxycholic acid in the treatment of intrahepatic cholestasis of pregnancy. A 12-year experience. Liver Int. 2005;25(3):548-54. doi: 10.1111/j.1478-3231.2004.0996.x. [PubMed:15910492].

14. Glantz A, Marschall HU, Lammert F, Mattsson LA. Intrahepatic cholestasis of pregnancy: a randomized controlled trial com- paring dexamethasone and ursodeoxycholic acid. Hepatology. 2005;42(6):1399-405. doi:10.1002/hep.20952. [PubMed: 16317669].

15. Kondrackiene J, Beuers U, Kupcinskas L. Efficacy and safety of ursodeoxycholic acid versus cholestyramine in intrahepatic cholestasis of pregnancy. Gastroenterology. 2005;129(3):894-901. doi:10.1053/j.gastro.2005.06.019. [PubMed:16143129].

16. Frezza M, Centini G, Cammareri G, Le Grazie C, Di Padova C. S adenosylmethionine for the treatment of intrahepatic cholestasis of pregnancy. Results of a controlled clinical trial. Hepatogastroenterology. 1990;37 Suppl 2:122-5. [PubMed: 2083923].

17. Nicastri PL, Diaferia A, Tartagni M, Loizzi P, Fanelli M. A randomised placebo-controlled trial of ursodeoxycholic acid and Sadenosylmethionine in the treatment of intrahepatic cholestasis of pregnancy. Br J Obstet Gynaecol. 1998;105(11):1205-7. [PubMed: 9853771].

18. Binder T, Salaj P, Zima T, Vitek L. Randomized prospective comparative study of ursodeoxycholic acid and S-adenosyl-L-methionine in the treatment of intrahepatic cholestasis of pregnancy.J Perinat Med. 2006;34(5):383-91. doi:10.1515/JPM.2006.077. [PubMed: 16965225].

19. Zhang L, Liu XH, Qi HB, Li Z, Fu XD, Chen L, et al. Ursodeoxycholic acid and S-adenosylmethionine in the treatment of intrahepatic cholestasis of pregnancy: a multi-centered randomized controlled trial. Eur Rev Med Pharmacol Sci. 2015;19(19):3770-6. [PubMed: 26502869].

20. Zhou F, Gao B, Wang X, Li J. Meta-analysis of ursodeoxycholic acid and S-adenosylmethionine for improving the outcomes of intrahepatic cholestasis of pregnancy [in Chinese]. Zhonghua Gan Zang Bing Za Zhi. 2014;22(4):299-304. doi: 10.3760/cma.j.issn.10073418.2014.04.013. [PubMed: 25173231].

21. Kenyon AP, Girling JC. Obstetrics Cholestasis. London: Royal College of Obstetricians and Gynaecologist; 2011.

22. Jadad AR, Moore RA, Carroll D, Jenkinson C, Reynolds DJ, Gavaghan DJ, et al. Assessing the quality of reports of randomized clinical trials: is blinding necessary?. Control Clin Trials. 1996;17(1):1-12. [PubMed: 8721797].

23. Kjaergard LL, Villumsen J, Gluud C. Reported methodologic quality and discrepancies between large and small randomized trials in meta-analyses. Ann Intern Med. 2001;135(11):982-9. [PubMed 11730399].

24. Moher D, Pham B, Jones A, Cook DJ, Jadad AR, Moher M, et al. Does quality of reports of randomised trials affect estimates of intervention efficacy reported in meta-analyses?. Lancet. 1998;352(9128):609-13. doi: 10.1016/S0140-6736(98)01085-X. [PubMed: 9746022].

25. DerSimonian R, Laird N. Meta-analysis in clinical trials. Control Clin Trials. 1986;7(3):177-88. [PubMed: 3802833].

26. Shadish WR, Haddock CK. Combining estimates of effects size. In: Cooper H, Hedges LV,ed. The handbook of research synthesis. New York: Russel Sage Foundation; 1994. pp. 261-81.

27. Floreani A, Paternoster D, Melis A, Grella PV. S-adenosylmethionine versus ursodeoxycholic acid in the treatment of intrahepatic cholestasis of pregnancy: preliminary results of a controlled trial. Eur J Obstet Gynecol Reprod Biol. 1996;67(2):109-13. [PubMed: 8841797].

28. Roncaglia N, Locatelli A, Arreghini A, Assi F, Cameroni I, Pezzullo JC, et al. A randomised controlled trial of ursodeoxycholic acid and Sadenosyl-1-methionine in the treatment of gestational cholestasis. BJOG. 2004;111(1):17-21. [PubMed: 14687046].

29. European Association for the Study of the Liver . EASL Clinical Practice Guidelines: management of cholestatic liver diseases. J Hepatol. 2009;51(2):237-67. doi: 10.1016/j.jhep.2009.04.009. [PubMed: 19501929].

30. Azzaroli F, Turco L, Lisotti A, Calvanese C, Mazzella G. The pharmacological management of intrahepatic cholestasis of pregnancy. Curr Clin Pharmacol. 2011;6(1):12-7. [PubMed: 21352094].

31. Beuers U. Drug insight: Mechanisms and sites of action of ursodeoxycholic acid in cholestasis. Nat Clin Pract Gastroenterol Hepatol. 2006;3(6):318-28. doi:10.1038/ncpgasthep0521. [PubMed: 16741551]. 
32. Almasio P, Bortolini M, Pagliaro L, Coltorti M. Role of S-adenosylL-methionine in the treatment of intrahepatic cholestasis. Drugs. 1990;40 Suppl 3:111-23. [PubMed: 2081476].
33. Lammert F, Marschall HU, Glantz A, Matern S. Intrahepatic cholestasis of pregnancy: molecular pathogenesis, diagnosis and management. J Hepatol. 2000;33(6):1012-21. [PubMed: 11131439]. 